\title{
Diagnostic accuracy of Bronchoalveolar lavage fluid in diagnosis of lung cancers in a tertiary care hospital in coastal region of Karnataka
}

\author{
Medha S. ${ }^{1}$, Sunil Kumar Y. ${ }^{2}$, Shetty K.P. ${ }^{3}$, Shetty J. \\ ${ }^{1}$ Dr. Medha Shankarling, Assistant Professor, ${ }^{2}$ Dr. Sunil Kumar Y, Professor, ${ }^{3}$ Dr. Shetty K Padma, Professor, \\ ${ }^{4}$ Dr. Shetty Jayaprakash, Professor and Head, all authors are affiliated with Department of Pathology, K S Hegde Medical \\ Academy, Mangalore, Karnataka 575018, India.
}

Address for Correspondence: Dr. Sunil Kumar Y, Professor, Department of Pathology, K S Hegde Medical Academy, Mangalore. E-mail id: drsunilkumary@rediffmail.com

\begin{abstract}
Introduction: Bronchoalveolar lavage (BAL) fluid analysis helps in early detection, rapid diagnosis and treatment of lung cancer as the therapy is based on subtyping. BAL has an important role in diagnosis of peripherally situated bronchoscopically invisible primary lung cancers, most of which are adenocarcinomas. Purpose: This study aims to detect diagnostic accuracy of BAL fluid analysis in detection of lung cancers. Materials and Methods: This retrospective cum prospective study in a tertiary care hospital involved analysis of BAL fluid obtained by lavage of respiratory tract in clinically and radiologically suspected lung lesions for 4 years from January 2012 to December 2016. Results: Out of 169 BAL fluids received, 38 (22.4\%) were positive for malignancy. Squamous cell carcinoma is the most common cancer noted in our study. BAL was reported falsely negative for malignancy in 49 cases (56.7\%) proved by lung biopsy. BAL was reported falsely negative for malignancy in 49 cases $(56.7 \%)$ proved by lung biopsy. One false positive case was noted in our study. The sensitivity of BAL was $43 \%$ and specificity of BAL was $98.8 \%$ in our study. The positive predictive value of BAL in the diagnosis of lung cancers is $97.36 \%$. The negative predictive value of BAL in the diagnosis of lung cancers is $62.59 \%$. The diagnostic accuracy of BAL is $70.4 \%$. Conclusion: BAL fluid analysis provides a rapid, reliable process to detect, subtype malignancies of the lower respiratory tract both in bronchoscopically visible and invisible tumours.
\end{abstract}

Keywords: Bronchoalveolar lavage, Cytology of lung, Diagnostic accuracy, Endobronchial growth, Lung cancer

\section{Introduction}

Cancers are the most common cause of death worldwide surpassing coronary heart disease and stroke. Lung cancer is the most common cancer in the world. It is associated with high incidence and high case fatality rate [1]. Lung cancer is estimated to be accounting for around $15 \%$ of newly detected cancers in India, more commonly in males, majorly attributed to smoking. There is increasing incidence of adenocarcinomas of lung in recent years [2]. Bronchoalveolar lavage (BAL) fluid analysis helps in early detection, rapid diagnosis and treatment of lung cancer as the therapy is based on subtyping. Small cell cancers are primarily treated with chemoradiotherapy

Manuscript received: $24^{\text {th }}$ August 2017

Reviewed: $4^{\text {th }}$ September 2017

Author Corrected: $11^{\text {th }}$ September 2017

Accepted for Publication: $18^{\text {th }}$ September 2017
[3]. Non-small cell lung carcinomas are treated with surgical resection for cure or palliative treatment with chemotherapy and radiotherapy [4]. BAL has an important role in diagnosis of peripherally situated bronchoscopically invisible primary lung cancers, most of which are adenocarcinomas [5]. Bronchoalveolar lavage (BAL) has both diagnostic and therapeutic uses. Analysis of BAL helps in the diagnosis of various inflammatory diseases and neoplasms of the lower respiratory tract. It helps in the detection of bacterial, fungal, parasitic and viral infections affecting the lung. BAL fluid can be used to detect occupational lung diseases, sarcoidosis, lung graft rejection and pulmonary lithiasis [6]. Diagnostic and prognostic markers can be used on BAL fluids that speed up the diagnosis. BAL specimens may also be used for 
molecular analyses in the search for diagnostic or prognostic markers [7]. The sensitivity of BAL is similar to transbronchial FNAC [8, 9]. BAL analysis has thus low morbidity and high diagnostic value [9].

This study conducted at a tertiary care hospital in coastal Karnataka attempts to analyze the diagnostic accuracy of BAL in diagnosis of biopsy proven lung cancers.

\section{Materials and Methods}

Type of study: This retrospective cum prospective study done for 4 years from January 2012 to December 2016.

Place of study: The study was carried out in a tertiary care hospital in a coastal region of Karnataka.

Sample collection: The study involved analysis of BAL fluid obtained by lavage of respiratory tract in clinically and radiologically suspected lung lesions for
4 years from January 2012 to December 2016. The BAL fluid thus obtained has been processed within one hour of collection. The BAL fluid was centrifuged at $3000 \mathrm{rpm}$ for 15 minutes. Four smears were made from the sediment. Two smears were stained with Leishman stain and the other two with Pap stain. Additional cytospin was used in case of low cellularity. The results of BAL were correlated with lung biopsy in all the cases. The biopsies are stained with H\&E stain and commented based on the morphology of cells.

Inclusion criteria: All the BAL fluid samples reaching pathology laboratory from patients admitted in our hospital were included in the study.

Exclusion criteria: All the BAL fluid samples reaching pathology laboratory outsourced from patients admitted in other hospitals were excluded from the study.

Statistical methods: $\mathrm{p}$ value was calculated by ANOVA using SPSS software.

\section{Results}

Out of 169 BAL fluids received, $38(22.4 \%)$ were positive for malignancy. There were 18 cases of squamous cell carcinomas (SCC), 15 cases of adenocarcinoma (AC), 4 cases of poorly differentiated carcinomas (PDCC) and 1 case of small cell carcinomas (SmCC) and 15 cases of severe dysplasia reported on BAL (Figures1-7). There were 36 cases of squamous cell carcinomas, 31 cases of adenocarcinomas, 3 cases of poorly differentiated carcinomas and 7 cases of small cell carcinomas and 9 cases of severe dysplasia reported on histopathology (chart 1). Squamous cell carcinoma is the most common cancer noted in our study. BAL was reported falsely negative for malignancy in 48 cases (56.7\%) proved by lung biopsy. The false negative cases in our study can be attributed to degenerated cells, presence of inflammation and interstitial malignancies with no endobronchial growth. Only one case of small cell carcinoma was detected on BAL out of seven biopsy proven cases. The small cell carcinoma cells have high nucleo-cytoplasmic ratio with scant cytoplasm that are usually mistaken for degenerated cells or inflammatory cells.

One false positive case was noted in our study. The dysplastic squamous cell on biopsy was falsely reported as malignancy on BAL. BAL was truly negative for malignancy in 82 cases (48.5\%), (Table 1). The sensitivity of BAL was $46 \%$ and specificity of BAL was $98.8 \%$ in our study. The positive predictive value of BAL in the diagnosis of lung cancers is $97.36 \%$. The negative predictive value of BAL in the diagnosis of lung cancers is $63 \%$.
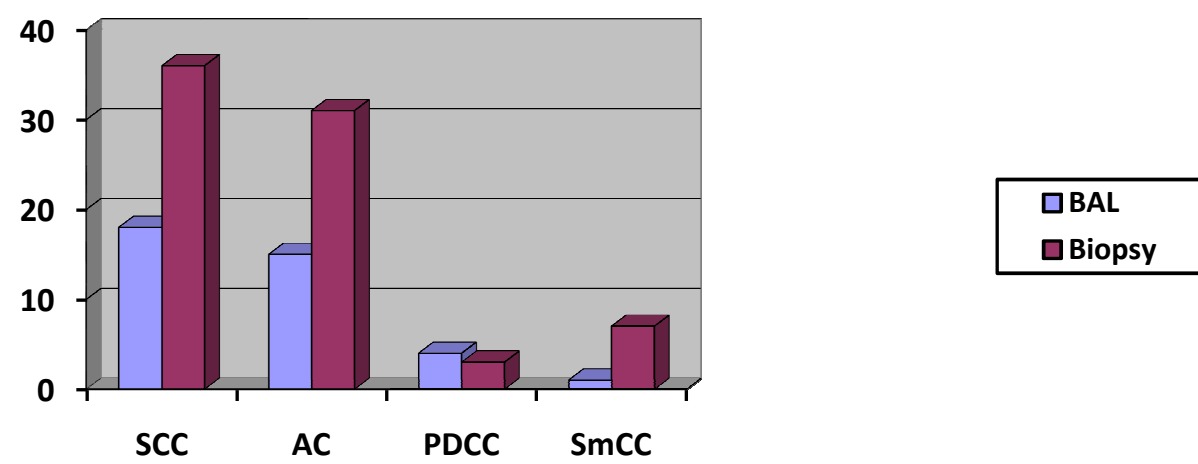

Chart-1: Comparison of diagnosis of lung cancer by BAL fluid analysis with biopsy. 
The diagnostic accuracy of BAL is $70.4 \%$. Majority of lung cancers were seen in males with male to female ratio being 4.73:1. The mean age of men was 60.8 years and of women was 58.4 years. The youngest patient was 30 years of age and the oldest was 88 years of age. All the bronchoscopically visible tumours were reported positive by BAL. BAL was positive for malignancy in one case $(1.1 \%)$ of bronchoscopically invisible tumours.

Table-1: Analysis of BAL fluid.

\begin{tabular}{|c|c|c|c|}
\hline & Lung cancer present & Lung cancer absent & Total \\
\hline BAL positive & 38 & 1 & 139 \\
\hline BAL negative & 48 & 82 & $\mathbf{1 6 9}$ \\
\hline Total & $\mathbf{8 6}$ & $\mathbf{8 3}$ & 130 \\
\hline
\end{tabular}

- Sensitivity $=46 \%$

- Specificity $=98.8 \%$

- Positive predictive value $=97.36 \%$

- Negative predictive value $=63 \%$.

- Diagnostic accuracy of BAL $=70.4 \%$.

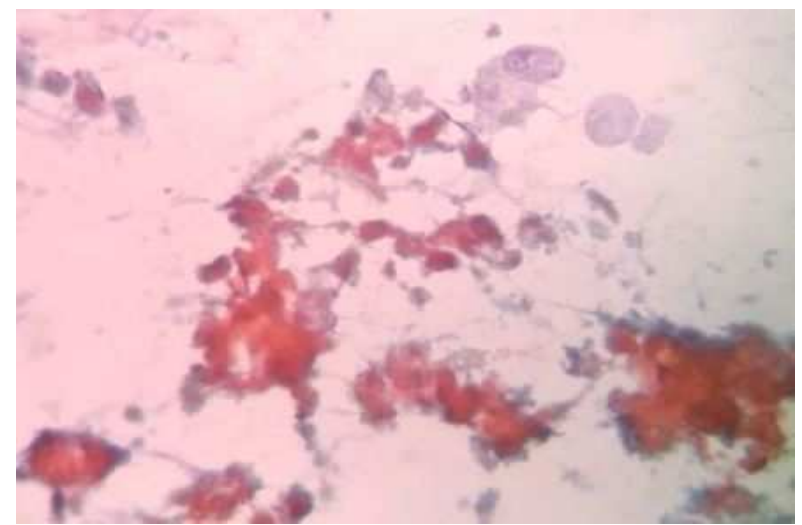

Figure-1: Squamous cell carcinoma displaying marked pleomorphism like high nucleocytoplasmic ratio, anisonucleosis, prominent nucleoli and dyskeratosis (Pap stain, high power, $x$ 45X)

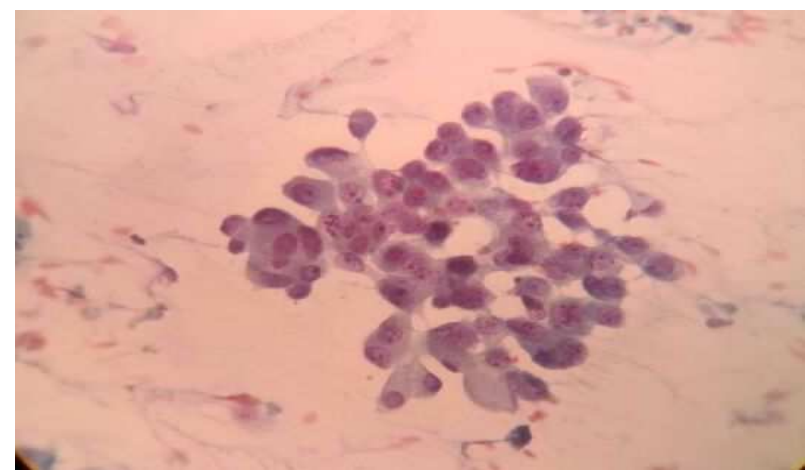

Figure-3: Adenocarcinoma displaying marked pleomorphism like high nucleocytoplasmic ratio, anisonucleosis, prominent nucleoli and vacuolated cytoplasm (Pap stain, high power, $\mathrm{x}$ 45X).

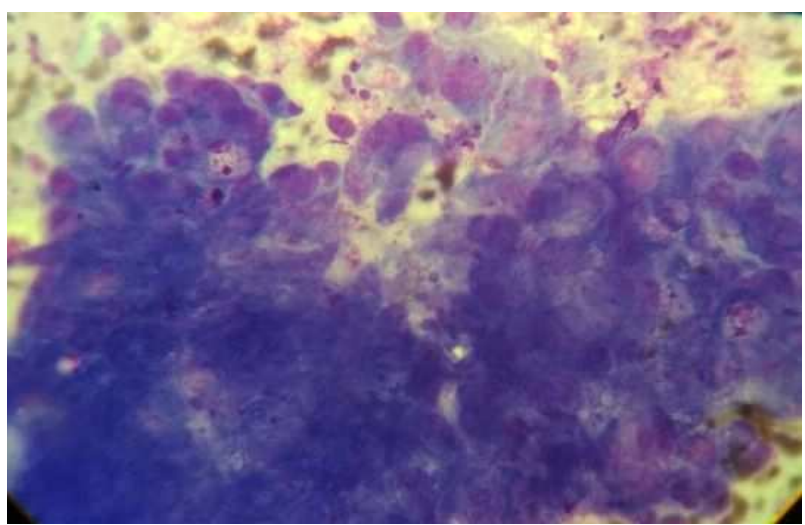

Figure-2: Squamous cell carcinoma displaying marked pleomorphism like high nucleocytoplasmic ratio, anisonucleosis and robin blue cytoplasm

(Leishman stain, high power, $\mathrm{x} 45 \mathrm{X}$ ).

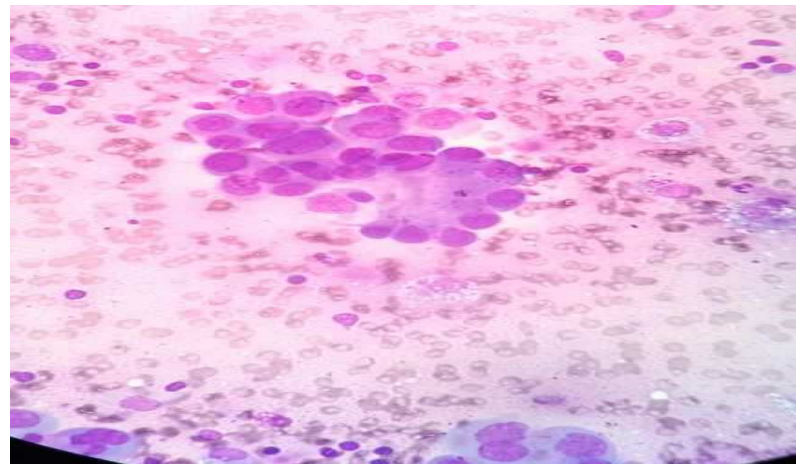

Figure-4: Adenocarcinoma displaying acinar pattern, fine granular chromatin, high nucleocytoplasmic ratio, anisonucleosis, prominent nucleoli and vacuolated cytoplasm (Leishman stain, high power, $x$ 45X). 
Research Article

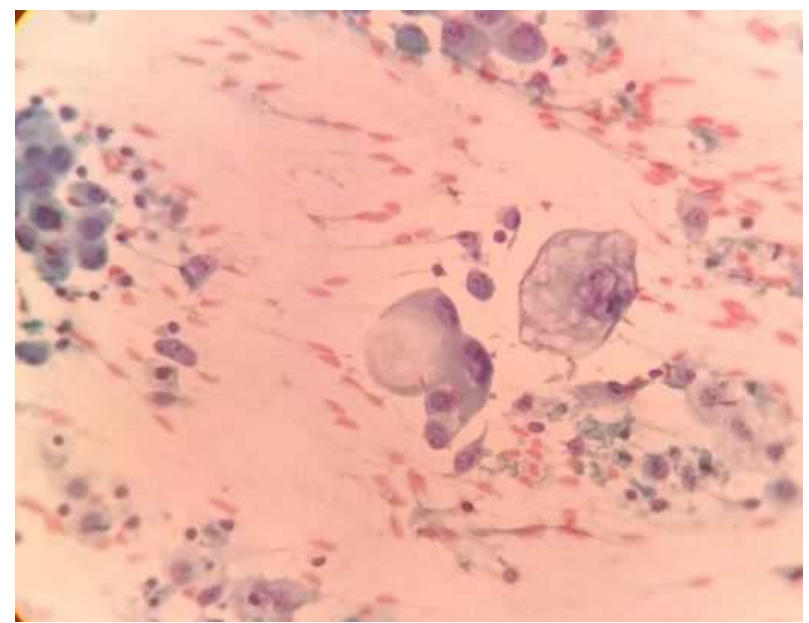

Figure-5: Adenocarcinoma displaying marked pleomorphism like high nucleocytoplasmic ratio, anisonucleosis, prominent nucleoli and vacuolated cytoplasm with signet ring appearance (Pap stain, high power, $\mathrm{x} 45 \mathrm{X}$ ).

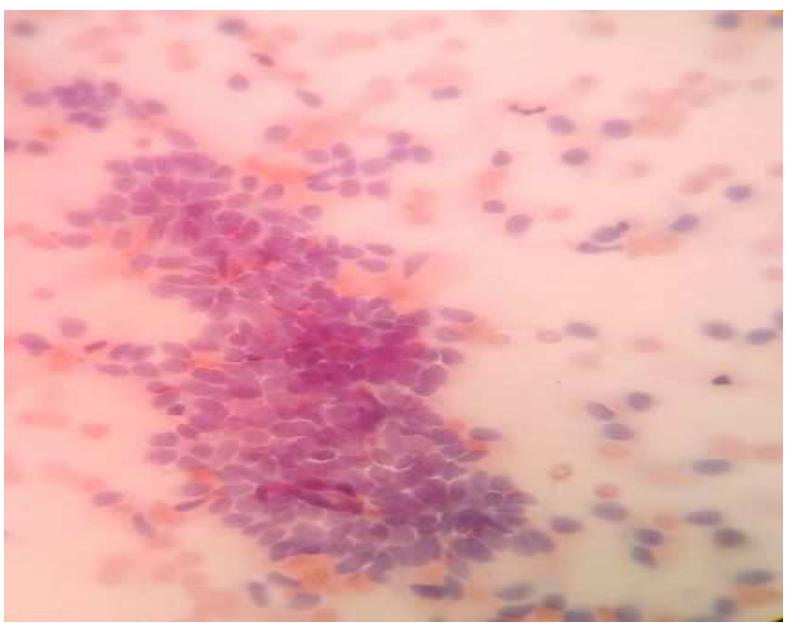

Figure-6: Small cell carcinoma displaying nuclear molding arranged in loosely cohesive clusters with scant cytoplasm and hyperchromatic nuclei (Pap stain, high power, $\mathrm{x}$ 45X).

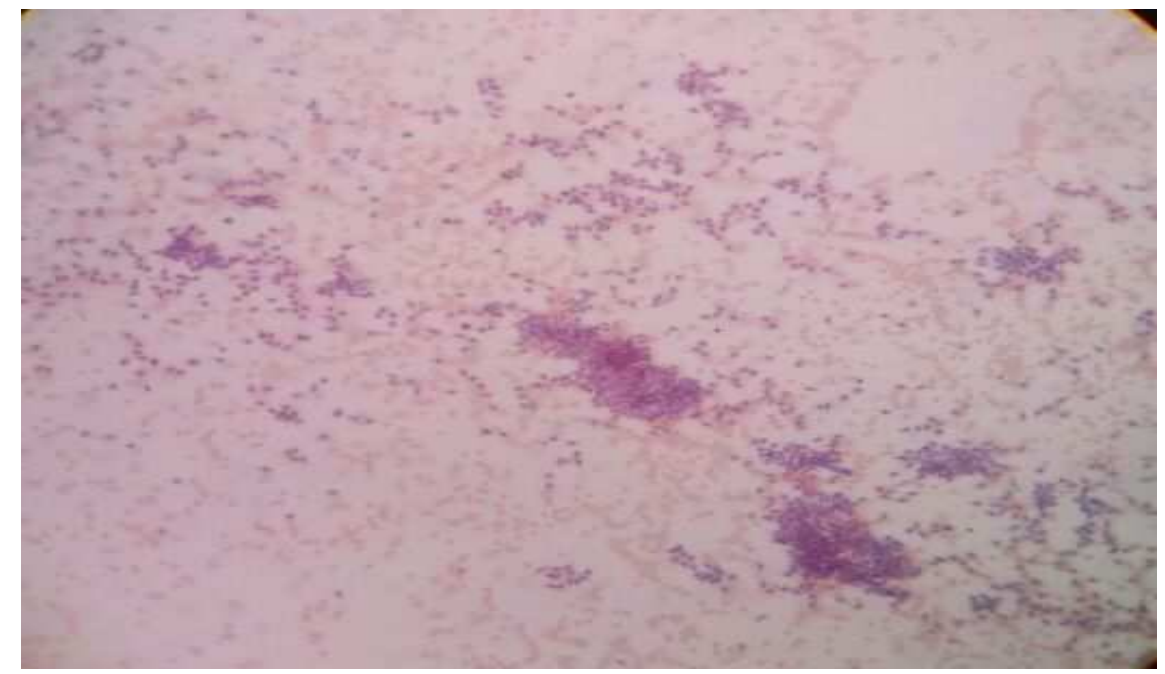

Figure-7: Small cell carcinoma displaying nuclear molding arranged in loosely cohesive clusters with scant cytoplasm and hyperchromatic nuclei (Pap stain, low power, $\mathrm{x} 10 \mathrm{X}$ ).

\section{Discussion}

A study by Linder et al [8] observed $68.6 \%$ accuracy of BAL in detection of malignancy. Gracia et al [10] found BAL accurate in 53\% cases. Piryozinski et al [9] found in $64.8 \%$ of cases BAL was accurate in diagnosing of lung cancer. The diagnostic accuracy of BAL was $70.4 \%$ in our study. Only one false positive case was noted in our study comparable to the study by Linder et al [8].

The diagnosis by BAL was in agreement with the biopsy in diagnosis of lung cancer in $79.1 \%$ and $35.9 \%$ cases in studies by Linder et al [8] and Piryozinski et al
[9] respectively. Our study found statistically significant agreement of BAL with biopsy proven lung cancers in $70.4 \%$ cases (chi square 58.13 and $\mathrm{p}$ value $<0.05$ ).

BAL provides information about the cells lining the respiratory tract in small bronchi that is beyond the reach of bronchoscope and bronchial brush. The accumulated secretion is aspirated initially followed by instillation and reaspiration of small aliquots of normal saline $(50 \mathrm{ml})$ from the selected bronchi. Analysis of BAL fluid is done on smears made from the sediment 
after centrifugation, which classifies the lesion as normal, inflammatory or neoplastic lung lesions [6].

BAL fluid from non neoplastic lesions yield endobronchial columnar epithelial cells, pigmented alveolar macrophages and mucus secreting goblet cells. Other cells like Clara cells, pneumocytes and neuroepithelial cells are rarely seen and can be identified only with special stains. The benign cells have smooth nuclear margins, even distribution of chromatin, basal nucleus surrounded by thin rim of cytoplasm. Terminal plate, cilia honeycomb pattern of arrangement of cells point towards benignity of the cells. Benign cells have small nucleoli as compared to adenocarcinoma cells with prominent nucleoli.

The alveolar macrophages contain ingested dust particles in their cytoplasm. The goblet cells are differentiated from signet ring cells of adenocarcinoma by the fact that the size and the nucleus of the goblet cell are similar to benign bronchial cell. The neoplastic signet ring cell is larger in size, nucleus is irregular and the cytoplasmic mucin causes indentation of the nucleus [6].

The BAL fluid may show mucin, amyloid, calcified deposits. A careful search for trapped cells in such cases helps in proper diagnosis. Ferruginous bodies in BAL fluid indicate considerable asbestos exposure [6].

Presence of drying artifact is identified by nuclear enlargement, loss of chromatin details, usually seen in the periphery of the smear, but there is uniform nucleus with preservation of nucleocytoplasmic ratio. Presence of contaminants during processing has to be identified. Presence of growth from BAL fluid on culture with minimal inflammatory cells is considered to be due to contamination [6].

Presence of multinucleated cells in BAL can be due to nonspecific reactive change due to trauma, injury, exposure to toxic fumes. However, tuberculosis, viral infections and sarcoidosis have to be ruled out [6].

The inflammatory lesions can show inflammatory cells like neutrophils in acute infections, lymphocytes and macrophages in chronic infections. Bacteria like streptococci, fungi like aspergillosis, can be commonly seen in BAL fluid. Our study had one case of aspergillus in BAL fluid. Presence of goblet cell hyperplasia is seen in COPD. Presence of eosinophils, Charcot Leyden crystals points towards bronchial asthma. Presence of alveolar calcospherites is seen in alveolar microlithiasis. BAL also plays an important role in diagnosis of sarcoidosis.

Increasing counts of neutrophils in BAL fluid helps in diagnosis of infection and rejection in lung transplant patients serving as a substitute for biopsy [6].

Basal cell hyperplasia can be a pitfall in diagnosis of small cell carcinoma. The benign basal cells are arranged in tight cohesive clusters, with smooth nuclear margins, uniformly distributed fine granular chromatin and scant cytoplasm. Nuclear moulding is absent in benign basal cells. In contrast, small cell carcinoma cells are loosely cohesive, display nuclear moulding, hyperchromatic nuclei and scant cytoplasm [6].

Squamous metaplasia may create a diagnostic confusion and is also found in increasing amounts in squamous cell carcinoma patients. Squamous metaplastic cells are arranged in cohesive clusters, with smooth nuclear margins.

Keratinization seen in the metaplastic squamous cells should be differentiated from keratinizing squamous cell carcinoma by the fact that nuclei are larger, darker and irregular in squamous cell carcinoma [6].

Radiation induced atypia is characterized by enlargement of the bronchial cells, with proportional enlargement of nucleus with preservation of nuclear architecture though there may be hyperchromasia and irregularity of nuclei can be seen [6].

Squamous cell carcinoma (SCC) cells in BAL fluid usually are in singles and loosely cohesive, with increased nucleocytoplasmic ratio, hyperchromatic angulated nuclei, irregular nuclear margins, dense homogenous cytoplasm and increased mitoses. Keratin is visualized as dense bright orangeophilic on Pap stain and robin blue colour on MGG stain. Squamous cell carcinoma can be classified into keratinizing and non keratinizing SCC [6].

Adenocarcinoma (AC) cells usually have raised nucleocytoplasmic ratio, fine granular chromatin, prominent nucleoli with moderate amount of vacuolated or mucinous cytoplasm [6]. 
Research Article

Small cell carcinoma cells are loosely cohesive and can be seen in singles with hyperchromatic nuclei, nuclear molding and scant cytoplasm. Small cell carcinoma should not be confused with basal cell hyperplasia and lymphocytes. Small cell carcinoma cell are 2-3 times larger than lymphocytes. The cells in cytology are usually classified as small, intermediate and large cells by comparing them to small lymphocytes. Small cells are 2-3 times the size of lymphocytes, intermediate cells are usually 3-4 times and large cells are 4-5 times the size of lymphocytes [6].

Pigments that can be seen in BAL fluid include carbon, hemosiderin, melanin and bile. Carbon pigment is seen in anthracosis. Hemosiderin laden macrophages can be seen in congestive heart failure, hemosiderosis. Bile is rarely seen in metastatic hepatocellular carcinoma.

Hemosiderin can be distinguished from brown melanin by its crystalline nature and blue colour development on Perl Prussian blue stain [6].

BAL fluid analysis helps to detect the bronchoscopically invisible tumours, tumours with lepidic growth pattern. One case in our study detected bronchoscopically invisible lesion but mass in the left upper lobe on CT scan. BAL helps early identification of dysplasia/ metaplasia when bronchoscopy is still unremarkable. There were fifteen cases of dysplasia noted in our study where bronchocopically no growth was seen. BAL also helps in identification of coexistent inflammatory/ infective conditions, collection of representative sample for microbial culture and antibiotic sensitivity.

Immunocytochemistry evaluation for markers like EGFR2 and ALK1 can be done on the BAL fluid to evaluate therapeutic use of monoclonal antibodies in palliative care of advanced lung cancers [6].

The pitfalls of BAL fluid analysis include rapidly occurring degenerative changes occurring within the exfoliated cells. The degeneration involves enlargement of cell, with enlarged nucleus, clumping of chromatin, vacuolation and disruption of the cytoplasm. Processing of BAL fluid within one hour of collection minimizes the degenerative changes occurring in the fluid. There were six cases with degenerated cells in our study. BAL does not detect parenchymal and interstitial lesions, but only intraalveolar and endobronchial lesions [6].

\section{Conclusion}

Our study is one of the few studies that are available on utility of BAL fluid analysis in detection of lung cancers. BAL fluid analysis provides a rapid, reliable process to detect, subtype malignancies of the lower respiratory tract both in bronchoscopically visible and invisible tumours. BAL also helps in collection of material for immunocytochemical evaluation of diagnostic and prognostic markers of lung malignancies.

\section{Contribution of Authors}

1. Medha Shankarling: data collection, reporting of slides, data analysis, preparation of article, proof reading.

2. Sunil Kumar Y: reporting of slides, data analysis, proof reading of article.

3. Shetty K Padma: reporting of slides, proof reading of article.

4. Shetty Jayaprakash: reporting of slides.

Funding: Nil, Conflict of interest: None initiated, Permission from IRB: Yes

\section{References}

1. Ferlay, J., Soerjomataram, I., Dikshit, R., Eser, S., Mathers, C., Rebelo, M., et al. Cancer incidence and mortality worldwide: Sources, methods and major patterns in GLOBOCAN 2012. Int. J. Cancer, 136 (5): E359 - E86. doi:10.1002/ijc.29210.

2. Behera D, Balamugesh T. Lung cancer in India. Indian J Chest Dis Allied Sci. 2004 Oct-Dec;46(4); 269-81.

3. Murray N, Coy P, Pater JL, Hodson I, Arnold A, Zee BC, Payne D, Kostashuk EC, Evans WK, Dixon P, et al. Importance of timing for thoracic irradiation in the combined modality treatment of limited-stage small-cell lung cancer. The National Cancer Institute of Canada Clinical Trials Group. J Clin Oncol. 1993 Feb;11 (2): 336-44.

4. Molina JR, Yang P, Cassivi SD, Schild SE, Adjei AA. Non-small cell lung cancer: epidemiology, risk factors, treatment, and survivorship. InMayo Clinic Proceedings 2008. Elsevier. May 3; 83 (5): 584-94. 
5. Wongsurakiat $\mathrm{P}$, Wongbunnate S, Dejsomritrutai W, Charoenratanakul S, Tscheikuna J, Youngchaiyud P, Pushpakom R, Maranetra N, Nana A, Chierakul N, Sakiyalak U, Ruengjam C. Diagnostic value of bronchoalveolar lavage and postbronchoscopic sputum cytology in peripheral lung cancer. Respirology. 1998 Jun; 3 (2):131-7.

6. Koss, Leopold G, Melamed, Myron R. Koss' Diagnostic Cytology and Its Histopathologic Bases, 5th ed. Lippincott, Williams and Wilkins, 2006: 646.

7. Tockman MS, Mulshine JL, Piantadosi S, et al. Prospective detection of preclinical lung cancer: Results from two studies of heterogeneous nuclear ribonucleoprotein A2/B1 overexpression. Clin Cancer Res 1997; 3:2237-46.
8. Linder J, Radio SJ, Robbins RA, Ghafouri M, Rennard SI. Bronchoalveolar lavage in the cytologic diagnosis of carcinoma of the lung. Acta Cytol. 1987 Nov-Dec;31(6):796-801.

9. Pirozynski M. Bronchoalveolar lavage in the diagnosis of peripheral, primary lung cancer. Chest. 1992 Aug; 102 (2): 372-4.

10. De Gracia J, Bravo C, Miravitlles M, Tallada N, Orriols R, Bellmunt J, Vendrell M, Morell F. Diagnostic value of bronchoalveolar lavage in peripheral lung cancer. American Review of Respiratory Disease. 1993 Mar 1; 147 (3): 649.

\section{How to cite this article?}

Medha S, Sunil Kumar Y, Shetty K.P, Shetty J. Diagnostic accuracy of Bronchoalveolar lavage fluid in diagnosis of lung cancers in a tertiary care hospital in coastal region of Karnataka. Trop J Path Micro 2017;3(3):322-328.doi: 10.17511/jopm.2017.i3.17. 